\title{
Questes
}

vestes Revue pluridisciplinaire d'études médiévales

$20 \mid 2011$

Maris et femmes

\section{Maris et femmes : avant-propos}

\section{Didier Lett}

\section{OpenEdition}

\section{Journals}

\section{Édition électronique}

URL : https://journals.openedition.org/questes/1803

DOI : 10.4000/questes.1803

ISSN : 2109-9472

\section{Éditeur}

Les Amis de Questes

\section{Édition imprimée}

Date de publication : 15 février 2011

Pagination : 1-11

ISSN : 2102-7188

\section{Référence électronique}

Didier Lett, « Maris et femmes : avant-propos », Questes [En ligne], 20 | 2011, mis en ligne le 01 janvier 2014, consulté le 26 août 2021. URL : http://journals.openedition.org/questes/1803 ; DOI : https:// doi.org/10.4000/questes.1803 


\title{
Avant-propos
}

\author{
Didier LETT \\ (Professeur, Université Denis Diderot, Paris VII)
}

Pour un médiéviste, le couple marital est au centre de toute réflexion sur la famille et la parenté. Au cœur de l'alliance entre deux familles, lieu de reproductions biologique et culturelle, il représente le noyau dur des structures de parenté et des structures familiales. De la qualité des relations entre mari et femme dépendent souvent celle des liens de parenté, de la gestion économique de l'entreprise familiale et de la transmission des valeurs et des modes de comportement à la génération suivante.

Étudier le lien marital, c'est s'intéresser aux relations entre deux « individus » de sexe différent au sein d'une union dont les normes sont devenues universelles dans le courant du XII ${ }^{\mathrm{e}}$ siècle. Face aux prescriptions juridiques et aux contraintes familiales, les maris et les épouses ont mis en pratique des modes de fonctionnement très divers selon les contextes sociaux et géographiques. Ce champ d'étude s'inscrit donc dans une histoire qui permet d'étudier l'individu et son rapport au groupe, l'identité, le genre, les émotions et l'articulation entre normes et pratiques, raisons pour lesquelles il est légitime qu'il fasse partie de la quête des jeunes historiens désireux de faire de l'histoire sociale.

L'interrogation sur le couple n'est pas neuve. Dans ce domaine, Georges Duby a été sans conteste l'un des pionniers en proposant dès 1973 de s'intéresser, dans son séminaire du Collège de France, à «Famille et sexualité au Moyen Âge » et en publiant, en 1981, Le Chevalier, la femme et le prêtre ${ }^{1}$. Par la suite, de très nombreuses études ont été produites sur le

\footnotetext{
${ }^{1}$ Georges DuBy, Le Chevalier, la femme et le prêtre, Paris, Gallimard, 1981.
} 
mariage, son évolution et ses rituels ou encore sur les échanges patrimoniaux liés à cette union. Aujourd'hui, à partir d'une documentation beaucoup plus diversifiée où les sources juridiques sont abondamment sollicitées, on tente de pénétrer plus avant dans les pratiques et l'intimité du couple, en centrant davantage l'attention sur 1'autonomie et la singularité des acteurs, en multipliant les études de cas $^{2}$. C'est dans cette nouvelle configuration qu'il convient de réinscrire ce nouveau bulletin de Questes dans lequel les auteurs ont choisi de délaisser le moment où le couple se forme pour s'intéresser davantage à la vie maritale avec ses amours, ses haines et, parfois, ses brutalités et ses séparations.

Avant que le lecteur puisse prendre connaissance des riches contributions qui suivent, je rappellerai quelques points qui me paraissent essentiels pour comprendre les enjeux de l'histoire des maris et des femmes au Moyen Âge.

Il semble d'abord capital de ne jamais oublier qu'il s'agit d'une union asymétrique où l'un des deux conjoints, l'épouse, doit être soumise à son mari car elle est « fille d'Ève », c'est-à-dire née après dans l'ordre de la Création, à partir de l'homme, et responsable de la Chute, deux éléments qui justifient et légitiment son infériorité, sa négativité et sa soumission. Par rapport à son conjoint qui a été créé « à l'image et à la ressemblance de Dieu », elle est une copie de copie, un produit dérivé. Au début du XII siècle, Rainaud, prieur de Saint-Eloi à Paris le rappelle : «L'homme est formé à l'image de dieu (ymaginem dei), la femme est fabriquée à la ressemblance de l'homme (similitudinem hominis). L'homme est fait pour

\footnotetext{
2 À titre d'exemples et pour ne retenir que des éléments d'une actualité récente, cf. Martine Charageat, Mariage et discipline des genres. Couples en conflit et justice en Aragon à la fin du Moyen Âge ( $X V^{e}-X V I^{e}$ siècles), Paris, Publications de la Sorbonne, 2011 (à paraître) ou Couples devant les justices du Moyen Âge à l'époque contemporaine, colloque organisé par Claude GAUVARD et Alessandro STELLA les 11 et 12 octobre 2010 à l'EHESS et à l'Université de Paris I Panthéon Sorbonne.
} 
lui-même, la femme est créée pour aider l'homme ; c'est pourquoi elle lui est soumise par la loi naturelle $»^{3}$.

De cette forte asymétrie naissent des assignations à comportement plus ou moins intégrées par l'ensemble des acteurs des deux sexes et des mises en pratique de la vie en commun. Dans les statuts communaux de la fin du Moyen Âge, l'épouse, à cause de sa fragilitas et de son imbecillitas sexus, est une éternelle mineure. Les statuts précisent souvent que pour être valides, les contrats, les donations ou les testaments souscrits par une femme mariée doivent être rédigés en présence et avec le consentement du mari. On peut ainsi lire dans les statuts de Esanatoglia (Marches) datés de 1324: «Nous statuons qu'aucune femme mariée ne puisse accuser, dénoncer ou déclencher une procédure inquisitoire à propos d'un méfait ou d'une faute sans le consentement exprès de son mari ou de son père si elle a un père et non un mari $»^{4}$.

Ces fortes assignations sont particulièrement visibles dans les conseils pédagogiques que les maris adressent à leurs jeunes femmes ou que les pères professent à l'égard de leurs filles bientôt en âge de se marier. Tous insistent sur la nécessaire soumission de l'épouse. On connaît les recommandations du Mesnagier de Paris, riche bourgeois anonyme, d'âge mur, qui rédige, vers 1393, un traité de morale et d'économie domestique destiné à sa très jeune épouse. Il lui rappelle souvent son devoir impératif de subordination, condition de la survie du couple. "Grâce à l'obéissance, écrit-il, une prudefemme gagne l'amour de son mari et finit par avoir de lui ce qu'elle désire. Mais je peux aussi affirmer que si vous décidez de ne pas obéir ou de vous montrer arrogante, vous vous perdez, vous-même, votre

\footnotetext{
${ }^{3}$ Paris, BnF, ms. lat. 2493, fol. 21vº cité par Emmanuel BAIN, " "Homme et femme il les créa" (Gen. 1, 27). Le genre féminin dans les commentaires de la Genèse au XII siècle », Studi medievali, fasc. 1 (juin 2007), p. 241.

${ }^{4}$ Gli statuti del comune di San Anatolia del 1324, Ancône, Gino Luzzato éd., 1909, Rub. CLVI, p. 102.
} 
mari et votre ménage $»^{5}$. On peut également rappeler que le Chevalier de la Tour Landry dans son fameux Livres pour l'enseignement de mes filles, rédigé en 1371-1372, consacre neuf chapitres sur 128 à l'obéissance et à l'humilité de l'épouse à l'égard de son mari ${ }^{6}$.

La domination masculine au sein du couple est souvent renforcée par un fort écart d'âge car, dans de nombreuses régions d'Occident, l'homme se marie à un âge bien plus élevé que la femme et il n'est pas rare que des écarts de dix ou quinze ans (sans parler des remariages) existent entre époux. Le vocabulaire utilisé pour désigner le mari, dans les termes de référence comme dans les termes d'adresse, est révélateur de son pouvoir de commandement. Il est le « chef de l'hostel», le «baron» ou le «sire». L'étude de la manière dont la femme est présentée et/ou décline son identité est également un signe probant de sa dépendance. Elle est désignée par un terme qui la subordonne à un homme : au cours de son cycle de vie, elle est «fille de », «épouse de », «mère de », « veuve de ». Son statut, étroitement lié à sa position au sein de la parenté, est strictement relatif. L'homme, quant à lui, n'est qu'exceptionnellement désigné par «mari de ». La manière de penser le monde est androcentrée et les épouses ne sont que les auxiliaires de ceux qui appartiennent aux ordres de la société. Au début du XII ${ }^{\mathrm{e}}$ siècle, l'évêque Gilbert de Limerick déclare : «Je ne dis pas que la fonction des femmes est de prier, de labourer ou de combattre, mais elles sont mariées à ceux qui prient, labourent et se battent, et les servent $»^{7}$.

${ }^{5}$ Le Mesnagier de Paris, texte édité par Georgina E. BRERETON et Janet M. FerRIER, traduction et notes par Karin UELTSCHI, Paris, Le Livre de Poche, «Lettres gothiques », 1994, p. 233-235.

${ }^{6}$ GeOFFroI DE LA TOUR LANDRY, Le Livre du Chevalier de La Tour Landry pour l'enseignement de ses filles, Anatole de MonTAIGLON (éd.), Paris, P. Jeannet, 1854.

${ }^{7}$ De statu ecclesiae, P L, 159, 997, cité par Georges DuBY, Les Trois Ordres, rééd. in Féodalité, Paris, Gallimard, « Quarto », 1996, p. 752-753. 
On retrouve cette forte asymétrie dans la manière dont est considéré l'adultère. Les lettres de rémission du règne de Charles VI présentent ce délit comme un crime féminin à $80 \%^{8}$. À Saragosse, au $X^{\mathrm{e}}$ siècle, le terme «adultère » n'est pas employé pour les hommes mais uniquement pour les femmes ${ }^{9}$. On peut reprocher à ces derniers d'avoir une concubine mais pas de pratiquer un acte adultère. En trompant son mari, l'épouse commet un péché charnel. En cocufiant sa femme, l'époux porte atteinte au sacrement de mariage et commet une faute spirituelle ${ }^{10}$. On ne peut donc analyser la vie du couple, sa normativité et ses déviances sans garder en mémoire la forte dissymétrie entre les sexes et la conception que, dès l'origine, la femme est du côté du caro et l'homme du côté de l'anima.

Ces normes récurrentes tentent de créer des pratiques mais ces dernières peuvent parfois être très éloignées des attentes masculines. Le thème littéraire du mari battu ou cocu est le signe d'une inversion jugée scandaleuse qui attire le charivari sous sa forme la plus déshonorante : la course de l'âne (l'asnade) au cours de laquelle l'homme ridiculisé chevauche dans les rues de la ville ou du village, le visage tourné vers la queue de l'animal ${ }^{11}$. Les fabliaux, à leur manière, en jouant sur l'inversion inacceptable des rôles, révèlent les peurs et les angoisses des hommes face au pouvoir potentiel des épouses. Ainsi, l'auteur de La Dame Ecouillée met

${ }^{8}$ Claude GaUvarD, «Honneur de femme et femme d'honneur en France à la fin du Moyen Âge », Francia, 28/1 (2001), p. 176.

9 À Saragosse au XV $\mathrm{XV}^{\mathrm{e}}$ siècle, seules les femmes sont accusées d'adulterium. Pour les hommes, on utilise un terme générique, celui d'amancebamiento, qui renvoie au concubinage. Iñaki BAZAN DIAZ, Delincuencia y criminalidad en el País Vasco en la transición de la Edad Media a la Moderna, Vitoria, 1995, p. 280.

${ }^{10} \mathrm{Cf}$. Martine ChARAGEAT, «La confrontation des genres au tribunal au Moyen Âge $\left(\mathrm{XIV}^{\mathrm{e}}-\mathrm{XVI}^{\mathrm{e}}\right.$ siècles). Une relecture des relations de couples en conflit», Genre \& Histoire, $\mathrm{n}^{\circ} 5$ (automne 2009), http://genrehistoire.revues.org/index775.html, paragraphe 14.

${ }^{11}$ Jean-Marie CARBASSE, " "Currant nudi". La répression de l'adultère dans le Midi médiéval », in Jacques POUMAREDE et Jean-Pierre RoYER (dir.), Droit, histoire et sexualité, Lille, Publications de l'Espace juridique, 1987, p. 83-102. 
en scène une femme dominatrice qui ne cesse de contredire son mari. Mais grâce à l'autorité du nouveau gendre, les hommes procèdent à une castration symbolique de l'épouse. Munis des testicules d'un taureau et d'un rasoir, ils prennent la femme et, l'incisant au niveau du bas ventre, lui font croire qu'ils viennent de lui extraire des testicules de son corps, cause de sa domination au sein du couple. Lorsqu'elle se réveille, on lui crie : « Nous tenons l'orgueil qui vous faisait oser». La domination masculine repose donc sur une explication biologique, anatomique. Si elle se traduit chez l'homme en termes de pouvoir légitime, chez la femme, elle se manifeste par de «l'orgueil». Après cette castration, l'épouse retourne à son humilité « naturelle »; son gendre lui dit : «Vous serez désormais une humble personne $»^{12}$.

Le thème littéraire et iconographique de la bataille pour la culotte plaide également en faveur de l'existence d'un réel pouvoir des femmes au sein du couple ou du moins à la revendication d'une autorité domestique ${ }^{13}$. On sait aussi le rôle parfois central que l'épouse du maître joue dans l'atelier, véritable entreprise familiale où l'espace de travail se confond avec celui de la résidence. La femme du maître tient la comptabilité, éduque les apprentis mais doit attendre le décès de son époux pour accéder véritablement à un statut « viril ».

Le pouvoir masculin permet au mari de battre son épouse, même si l'ensemble des statuts ou des traités lui conseille en ce domaine la plus grande modération. La coutume en vigueur dans la vallée de Barèges (Pyrénées) en 1404 proclame : «Tout maître et chef de maison peut châtier femme et famille sans que nul ne puisse y mettre obstacle». Les statuts

12 La Dame Ecouillée, dans Contes pour rire? Fabliaux des XIII et XIV siècles, traduits par Nora ScOTT, Paris, 10-18, 1977, p. 193-209.

13 Pierre BUREAU, «La "dispute pour la culotte": variations littéraires et iconographiques d'un thème profane (XIII ${ }^{\mathrm{e}}-\mathrm{XVI}^{\mathrm{e}}$ siècles)», Médiévales, 29 (1995), p. 105-129. 
d'Apiro (Marches) en 1388 punissent l'homme qui a frappé son épouse, ses enfants ou ses domestiques uniquement s'il a utilisé une arme en $\mathrm{fer}^{14}$. La dureté maritale provoque parfois des conflits violents au sein du couple où le mari semble souvent le vainqueur ${ }^{15}$. La cellule conjugale serait-elle le lieu d'une plus forte subordination de la femme et de tensions plus vives entre les deux sexes, un lieu où la femme serait moins protégée par le droit ? Sans doute, mais il convient de faire attention aux effets de sources car nous savons bien que seules les affaires malheureuses parviennent aux historiens à travers les sources judiciaires. Pour un couple qui se bat et se déchire dans la documentation, combien se taisent ou s'aiment en silence ? $\mathrm{Au} \mathrm{XIII}^{\mathrm{e}}$ siècle, Etienne de Bourbon commence un exemplum ainsi : «Le diable avait travaillé pendant trente ans et plus à désunir deux époux, et n'avait pu même arriver à leur faire échanger une seule fois des paroles désagréables ${ }^{16}$.

Au Moyen Âge, les maris n'ont pas le monopole de la violence et du crime. Il existe aussi des épouses criminelles ${ }^{17}$ qui font tuer leur mari par un ou plusieurs hommes de main, telle Huguette de Sainte-Croix, dame de Saint-Laurent-la-Roche dans le Jura qui est accusée d'avoir exécuté son mari, Etienne de Saint-Dizier en février 1328 avec l'aide du propre frère de

\footnotetext{
${ }^{14}$ Gli statuti di Apiro dell'anno 1388, Dante CECCHI, Milan, A. Giuffre, 1984, Livre IV, VI, p. 153.

15 Parmi une littérature désormais abondante sur le sujet, on signalera: Richard $\mathrm{H}$. Helmholz, Marriage Litigation in Medieval England, Cambridge, Cambridge University Press, 1974 ; Frederik Pedersen, Marriage Disputes in Medieval England, Londres, Hambledon, 2000; Sarah M. ButLER, The Language of Abuse: Marital Violence in Later Medieval England, Leiden, Brill, 2007 ; Peter J. P. GolDBERG, "Gender and Matrimonial Litigation in the Church Courts in the Later Middle Ages: The Evidence of the Court of York », Gender and History, vol. 19, 1 (2007), p. 43-59.

${ }^{16}$ Paris, BnF, ms. lat. 15970, fol. 319 (Tubach 5361), cité dans Le Rire du prédicateur. Récits facétieux du Moyen Âge, textes traduits par Albert LECOY DE LA MARCHE, présentation, notes et annexes par Jacques BerLIOZ, Turnhout, Brepols, 1992, p. 132.

${ }^{17}$ Cf. Loïc CAdiet, Frédéric Chauvaud, Claude Gauvard, Pauline SchmitT PanTel, Myriam TsiKounas (dir.), Figures de femmes criminelles de l'Antiquité à nos jours, Paris, Publications de la Sorbonne, 2010.
} 
la victime, Guillaume, sire d'Alièze ${ }^{18}$ ou telle Blanche d'Overbreuc en mars 1449, incriminée dans le meurtre de son mari, Guillaume de Flavy, capitaine de Compiègne ${ }^{19}$. Ces haines ou ces impossibilités de poursuivre une vie commune ne se terminent pas toujours par des crimes et il arrive que des couples se séparent ${ }^{20}$.

Fort heureusement, l'historien peut également noter des sentiments d'amour et d'affection à l'intérieur de la cellule conjugale. C'est en tout cas, à la fin du Moyen Âge, un thème qui retient l'attention des prédicateurs. Parmi les sujets des nombreux sermons professés par Bernardin de Sienne en 1427, on rencontre : «Comment le mari doit aimer son épouse, et l'épouse son mari » ou « Du bon amour qu'il peut exister entre une femme et son mari » ou encore «Comment le mariage doit être considéré ». Si, dans le Décret de Gratien, l'affectio maritalis est plutôt synonyme de « consentement », elle revêt un sens nouveau à partir de la fin du XII ${ }^{\mathrm{e}}$ siècle et, dans les Décrétales d'Alexandre III, Clément III ou Innocent III, elle exprime une disposition active que les époux ont le devoir de cultiver, une conduite à tenir à l'intérieur du mariage qui se retrouve dans tous les milieux sociaux. Même au sein de la très haute aristocratie de la fin du Moyen Âge où pourtant les mariages sont si bien préparés pour

\footnotetext{
${ }^{18}$ Michelle BubeniCEK, Le Donjon, la femme et le beau-frère. Huguette de SainteCroix ; une épouse meurtrière ? Essai d'histoire totale d'un dossier à charge, mémoire inédit (volume III) dans le cadre d'une HDR soutenue en novembre 2010 à l'Université de Paris I Panthéon Sorbonne.

${ }^{19}$ Claude GAUVARD, «Entre justice et vengeance : le meurtre de Guillaume de Flavy et l'honneur des nobles dans le royaume de France au milieu du $\mathrm{XV}^{\mathrm{e}}$ siècle », in Jacques PAVIOT et Jacques Verger (dir.), Guerre, pouvoir et noblesse au Moyen Âge. Mélanges en l'honneur de Philippe Contamine, Paris, Presses de l'Université de Paris-Sorbonne, 2000, p. 291-311.

20 Cf. Diego Quaglioni et Silvana SEIDEL MENCHI (dir.), Coniugi nemici: la separazione in Italia dal XII al XVIII secolo, Bologne, Il Mulino, 2000 ; Matrimonio in dubbio : unioni controverse e nozze clandestine in Italia dal XIV al XVIII secolo, Bologne, Il Mulino, 2001 ; Emmanuelle SANTINELLI (dir.), Répudiation, séparation, divorce dans l'Occident médiéval, Valenciennes, Presses Universitaires de Valenciennes, 2007.
} 
des raisons stratégiques et diplomatiques, l'affectio maritalis existe, comme on peut l'analyser à travers les récits des premières rencontres entre princes et princesses en France et en Angleterre à la fin du $\mathrm{XIV}^{\mathrm{e}}$ et au $\mathrm{XV}^{\mathrm{e}}$ siècles ${ }^{21}$.

Cette affection conjugale ne se donne jamais aussi bien à voir que dans les rares sources épistolaires de la fin du Moyen Âge. L'abondante et fameuse correspondance entre Margaret et John Paston, famille de la gentry du Norfolk du milieu du $X V^{\mathrm{e}}$ siècle, évoque certes souvent la gestion du domaine mais n'en laisse pas moins filtrer un réel attachement entre les deux conjoints. Margaret se plaint des trop longues absences de son mari, John Paston, qui, selon elle, réside trop souvent à Londres. Les dernières phrases de ses lettres sont toujours chargées de grande tendresse : «Je vous prie instamment de me faire tenir des nouvelles de votre santé et de vos affaires, car, soyez sûr que je n'aurai pas de repos avant d'avoir de vos nouvelles » (15 février 1449) ; «Je vous prie, ne soyez pas avare de lettres d'ici votre retour. Je voudrais en recevoir une tous les jours...» (janvier $1453)$; « le temps me parait long tant que je n'ai pas de vos nouvelles » (13 mai 1465) etc. Les clauses testamentaires les plus longues sont très souvent celles qui sont consacrées à l'épouse, révélant de la part du mari un profond souci d'assurer à cette dernière les garanties maximales pour qu'elle puisse vivre décemment après son décès.

L'amour conjugal est aussi physique. Les prédicateurs et confesseurs de la fin de l'époque médiévale ont développé un abondant discours sur l'acte charnel. Ainsi Thomas de Chobham, dans son manuel de confesseurs (la Summa Confessorum) distingue trois types de coït conjugal. D'abord, le coït licite fait de rapports charnels voués à la procréation ou de ceux qui ont pour fonction d'éviter la fornication (relations extra-conjugales) en

\footnotetext{
${ }^{21}$ Manuel GuAY, «Du consentement à l'affectio maritalis : quatre mariages princiers
} (France-Angleterre, 1395-1468) », Revue Historique, n 650, 2 (2009), p. 291-319. 
respectant bien entendu les interdits liés au calendrier liturgique. Ensuite, le coït fragile, accompli sans contrôle de ses pulsions. Enfin, le coït impétueux qui recouvre trois types de rapports : ceux qui se produisent entre époux qui s'aiment trop, considérés comme une forme d'adultère car, en ce cas, les maris traitent leur femme comme des prostituées, s'unissant à elle par volupté, par recherche du plaisir et non uniquement pour procréer ; le coït contre-nature, non vaginal et vaginal n'adoptant pas la position du missionnaire (position révélatrice au sein de la sexualité médiévale de l'asymétrie évoquée plus haut); et enfin le coït qui se déroule à des moments non autorisés par l'Église ou pendant la grossesse, les menstrues et les relevailles de l'épouse ${ }^{22}$.

On sait que pour certains théologiens, tel Gratien, la consommation s'avère indispensable pour valider l'union matrimoniale. L'acte charnel licite est nécessaire et présenté par l'Église comme « une dette conjugale » (debitum conjugale) que les époux se doivent mutuellement. La sexualité conjugale s'avère donc un sujet central pour qui veut étudier l'histoire des couples $^{23}$. Mais, parce qu'on entre dans l'intimité la plus grande d'une société où la pudeur est si vivace, elle nous échappe en grande partie. Les sources permettant d'éclairer la vie sexuelle des couples au Moyen Âge, en effet, émane essentiellement d'un discours théorique. Lorsque l'on pénètre aussi intimement au plus profond de la famille, la documentation se dérobe. En 1359, une longue enquête est menée à Entrevennes en Haute Provence pour tenter de prouver le mariage de Sybille de Cabris après le décès de son époux Annibal de Moustiers face à sa belle famille qui cherche à récupérer des biens. Parmi les témoins interrogés, Raymonde Cabrilhana, amie et

22 Thomae de Chobham Summa Confessorum, F. BROOMFIELd (éd.), (Analecta Mediaevalia Namurcensia 25), Louvain, Éditions Nauwelaerts, 1968, VII, Questio IIa, De coitu coniugali, p. 333-339.

${ }^{23}$ Voir en dernier lieu l'ouvrage de Ruth Mazo KARRAS, Sexuality in Medieval Europe. Doing unto others, New York et Londres, Routledge, 2005. 
voisine du couple évoque la grande tendresse et l'affection qui liaient les deux époux : «Car j'ai bien souvent fréquenté leur maison, dit-elle, et j'ai fréquemment vu son mari l'embrasser. Je l'ai vue, à plusieurs reprises, dans un seul et même lit avec son mari, nue à nu, seule à seul. J'ai vu leur beauté, leur jeunesse et leur aptitude à se livrer aux gestes de la copulation. De ce que j'ai vu, je ne puis témoigner, mais vous pouvez m'en croire $»^{24}$. Elle s'arrête là ; n'en dira jamais plus. L'historien, comme souvent, reste sur sa faim.

${ }^{24}$ Noël CoulET, Affaires d'argent et affaires de famille en Haute Provence au XIV siècle. Le dossier du procès de Sybille de Cabris contre Matteo Villani et la compagnie des Buonaccorsi, Rome, Collection de l'École française de Rome-158, 1992, p. 76. 